\title{
\begin{tabular}{l|l} 
Mibraries & DSpace@MIT
\end{tabular}
}

\author{
MIT Open Access Articles
}

\section{A Perspective on the Revival of Structural (In) Stability With Novel Opportunities for Function: From Buckliphobia to Buckliphilia}

The MIT Faculty has made this article openly available. Please share how this access benefits you. Your story matters.

Citation: Reis, Pedro M. “A Perspective on the Revival of Structural (In) Stability With Novel Opportunities for Function: From Buckliphobia to Buckliphilia." Journal of Applied Mechanics 82.11 (2015): 111001 . (C) 2015 by ASME

As Published: http://dx.doi.org/10.1115/1.4031456

Publisher: ASME International

Persistent URL: http://hdl.handle.net/1721.1/110704

Version: Final published version: final published article, as it appeared in a journal, conference proceedings, or other formally published context

Terms of Use: Article is made available in accordance with the publisher's policy and may be subject to US copyright law. Please refer to the publisher's site for terms of use. 


\section{Pedro M. Reis \\ EGS. Lab: Elasticity, \\ Geometry, and Statistics Laboratory, Department of Mechanical Engineering, Massachusetts Institute of Technology, Cambridge, MA 02139 e-mail: preis@mit.edu}

\section{A Perspective on the Revival of Structural (In)Stability With Novel Opportunities for Function: From Buckliphobia to Buckliphilia}

Buckling of slender structures is traditionally regarded as a first route toward failure. Here, we provide an alternative perspective on a burgeoning movement where mechanical instabilities are exploited to devise new classes of functional mechanisms that make use of the geometrically nonlinear behavior of their postbuckling regimes. Selected examples are highlighted across length-scales to illustrate some of the exciting opportunities that lie ahead. [DOI: 10.1115/1.4031456]

\section{Introduction}

Slender structural elements (e.g., rods, plates and shells) under compression are ubiquitously subjected to mechanical instabilities. In 1744, Euler [1] laid the foundation for the formal analysis of structural stability; a field that has matured to become paramount in engineering design and one of the pillars in the history of mechanics [2]. A modern and detailed perspective on the stability of structures is found in the seminal book by Bažant and Cedolin [3]. Across length-scales, buckling has traditionally been regarded as a first route toward failure and can lead to catastrophic collapse [4]; an approach that can be succinctly referred to as Buckliphobia. By contrast, Buckliphilia is a more recent and burgeoning trend that is changing the above paradigm. Mechanical instabilities of slender structures are therefore envisioned as opportunities for novel modes of functionality that are to be predictively understood in order to then be exploited.

In these efforts of adopting Buckliphilia, there is a need to rationalize the complex configurations that arise in the postbuckling regime, far-from-threshold. The large displacements and rotations permissible by slender structures can yield nontrivial and nonnegligible geometric nonlinearities, even if their material properties remain linear. Furthermore, this strong rooting of the postbuckling behavior on geometry results in general and universal modes of deformation; albeit with threshold or onsets that are scale- or material-dependent. To avoid overstatement, it is important to note that viscoelasticity, plasticity, fracture, and other phenomena can introduce additional time- and length-scales that may compromise the geometric universality of the buckling modes. In many problems involving the large deformation of thin structures, however, the strains at the material level are small enough, such that these effects are secondary and a linear elastic constitutive description suffices.

Qualifying a structure as slender is a statement on aspect ratio rather than length-scale, which added to the fact that elasticity is a scale-free theory, ensures that functional mechanisms based on the instability of slender structures can be instantiated over a wide range of length-scales. In Fig. 1, we represent three representative examples that illustrate this scale invariance. First, the crumpling of paper [5] (Fig. 1(a)) has been regarded by the nonlinear physics community as a canonical problem, at the centimeter scale, for

Contributed by the Applied Mechanics Division of ASME for publication in the Journal OF ApPlied Mechanics. Manuscript received August 24, 2015; final manuscript received August 25, 2015; published online September 28, 2015. Editor: Yonggang Huang.
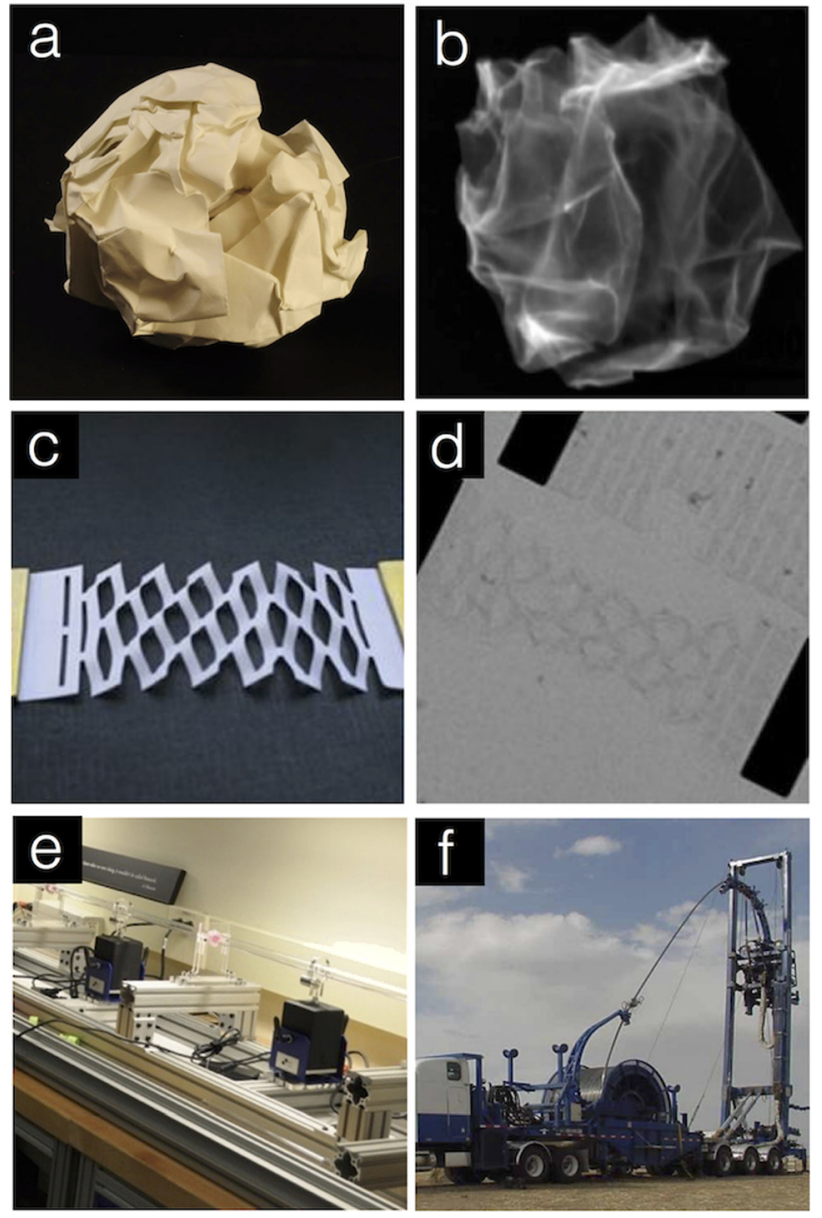

Fig. 1 Mechanics of slender structures, across length-scales. (a) Crumpled paper ball (8-cm diameter) and (b) crumpled graphene sheet ( $\sim 200-\mathrm{nm}$ diameter, SEM image courtesy of Mao et al. [9]). Kirigami springs made out of a periodically cut (c) paper and (d) graphene sheets (courtesy of Blees et al. [11]). (e) Precision desktop experiment to study the buckling of a thin rod injected into a horizontal cylindrical constraint that is applicable to the $(f)$ lock-up in coiled tubing operations in the oil and gas industries (courtesy of Miller et al. [14]). 
how elastic singularities (developable cones [6] and ridges [7]) can assemble into complex arrangements that require a description based on statistical mechanics [8]. Analogously, at the nanoscale, spheres of crumpled graphene, the epitome of a thin sheet, are being explored for innovative energy storage applications (Fig. 1(b)) [9,10].

As a second illustration of the scale-invariance in plates, in Figs. $1(c)$ and $1(d)$, we present two examples of kirigami, for paper and graphene sheets, respectively; a variation of origami that involves shape forming but allowing cutting [11]. Again, because of the preponderance of geometry, the modes of deformation in the paper and graphene cases are identical. Building on the insight gained by their paper models, Blees et al. [11] have devised a new class of graphene-based metamaterials with tunable mechanical properties, that may open new directions in stretchable electrodes, springs, and hinges.

In a third example of our own (Figs. 1(e) and 1(f)), we have performed precision model experiments (at the meter scale) [12-14] to study the buckling and subsequent lock-up of coiled-tubing during injection into horizontal wellbores (at the kilometer scale), as well as identify methods for extending reach [13]. As part of this study, we performed a formal scaling analysis of the dimensionless governing equations for a thin elastic rod inside a cylindrical constraint to identify the primary parameters and predictively extrapolate the mechanics learned from the scaled experiment to the field scale. This example illustrates the value of scalings in order to assist in the porting of the gained understanding across length-scales, in a predictive manner. Scaling analysis is therefore a powerful tool, even if the ultimate goal is to be able to arrive at an analytical or numerical quantitative description of the problem.

The hegemony of geometry and the scale-invariance of Buckliphilia requires that we revisit old problems on the mechanics of rods, plates, and shells and tackle the fundamental challenge of predictively understanding their underlying geometric nonlinearities in the postbuckling regime. We proceed by providing four specific examples of practical relevance, where this methodology can be used to derive function. A more detailed review of other instances of functional buckling can be found in Refs. [15 and 16].

Figure 2(a) shows a photograph of a pneumatically actuated structure that makes use of the periodic buckling of a thin-stiff shell bound to a thick-soft substrate to generate complex topography, on demand [17], by depressurizing an inner cavity. In a specific region of parameter space, one can excite a wrinkling pattern of dimples that arrange in a quasi-hexagonal packing, with topological defects that are dictated by the geometry of the curved crystal [18]. The depth of the dimples can be varied by setting the pressure differential between the inside and the outside of the sample. These dimpled patterns have a striking resemblance to the topography of golf balls. We have explored this similarity and demonstrated that the reversible buckling patterns on spherical shells can be exploited as a tunable and reversible aerodynamic drag reduction mechanism, which we refer to as Smorphs (for smart morphable surfaces) [17]. These Smorphs have the remarkable characteristic that their aerodynamic drag coefficient can be controlled and actively reduced by as much as a factor of 2 (from $C_{D} \approx 0.5$ for the smooth spherical case, down to $C_{D} \approx 0.25$ for the dimpled case). Moreover, we have been able to rationalize how the curvature of the substrate, together with the other geometric and material properties of the film and substrate, dictates the phase diagram of the system [19].

The Buckliball (Fig. 2(b)), was introduced as a new class of continuum structures that can undergo buckling-induced reversible folding, under pneumatic actuation [20]. The geometry of the Buckliball consists of a spherical shell patterned with a regular array of circular voids, the arrangements of which is dictated by topological constraints. Below a critical value of the internal pressure, the narrow ligaments between the voids buckle, leading to a cooperative cascade that is underpinned by a negative Poisson's ratio effect [21] of the skeleton of the ball. This mechanism can potentially be used for encapsulation, actuation in soft robotics [22], or for large-scale deployable structures.

In another example for the functional usage of buckling, this time at the microscopic scale, $\mathrm{Xu}$ et al. [23] recently proposed a new set of design and fabrication principles to morph planar layouts of ribbons into a plethora of three-dimensional (3D) architectures (Fig. 2(c)). These include butterflies, boxes, tents, tables, baskets, coils, and stars, with the possibility of built-in hierarchy. The 2D-to-3D transition is triggered by buckling that is induced by the compressive stresses arising from the unloading of a prestretched elastomeric substrate, onto which the ribbons are adhered to at strategic bounding sites. The mechanism is analogous to children's pop-up books and is related to the kirigami example mentioned above. Here, however, the coupling of bending and twist of the ribbons enables a wider design flexibility than those more traditional strategies, which are limited by the additional geometric constraints of plates. Potential applications of these 3D devices span novel electronic, optical and magnetic properties, which themselves may be coupled, and with the added advantage of the possibility of tunability.

Shifting to biological systems; still at the micron scale, uniflagellated bacteria (e.g., Vibrio alginolyticus, see Fig. 2(d)) have been found to exploit buckling to alter their swimming direction [24]. In this case, the instability is localized at the highly compliant hook that connects the slender flagellum to the rotary motor inside the cell body. Subsequently, by combining computer simulations and precision model experiments, we have demonstrated [25] that it is possible for the actual flagella to buckle, itself a helical filament that is highly flexible ought to its slenderness. Here, the instability arises due to the compressive stresses that derive from the viscous loading during the rotation of the helical filament. Given our dimensionless description in a problem that is, again, dominated by geometry, we have extrapolated these results to the biologically relevant regime and found that some common bacteria appear to operate near the critical buckling
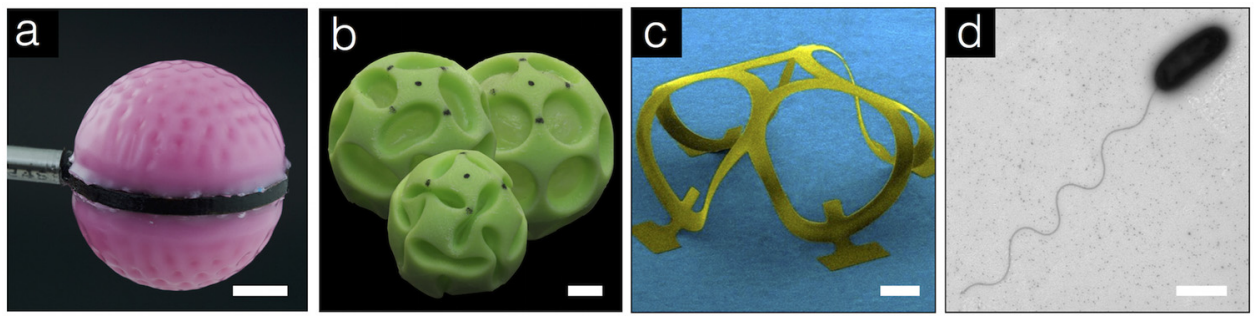

Fig. 2 Buckliphilia - using buckling for functional modes of deformation. (a) Smart morphable surfaces for tunable aerodynamic drag reduction through wrinkling on curved surfaces [17]. Scale bar, $1 \mathrm{~cm}$. (b) The Buckliball exhibits buckling-induced folding [20]. Samples at different stages of loading. Scale bar, $1 \mathrm{~cm}$. (c) 3D structure created by the buckling of a 2D ribbon layout (courtesy of Xu et al. [23]). Scale bar, $200 \mu \mathrm{m}$. (d) Vibrio alginolyticus uses buckling of its hook for turning during swimming (courtesy of Son et al. [24]). Scale bar, $1 \mu \mathrm{m}$. 
threshold. This raises the hypothesis that the reconfigurations that emerge in the postbuckling regime of the flagella may be used as a remarkably simple functional turning mechanism. We speculate that there are many other instances in biology, yet to be discovered and mimicked, where mechanical instabilities are used for function.

These new trends of reversibly exploiting the postbuckling regime of slender structures are calling for new technical approaches for experimental, numerical, and theoretical analysis.

On the experimental front, to which we dedicate particular focus in our research, scalability of the geometrically rooted behavior in thin structures allows for a reduction of problems to their bare essential physical ingredients. As such, precision model experiments can be devised at a scale (e.g., the centimeter) that is more amenable for systematic and detailed exploration, when compared to the length scale of the original problem (the micron or the kilometer, in many cases). These rescaled laboratory settings, which we often refer to humorously as precision desktop experiments (p.d.e.'s), carry added value in that they can serve as quantitative validation platforms for theoretical and numerical models, as well as a powerful means to gain physical insight. More importantly, the added versatility in fabrication and systematic exploration of parameter spaces open the door for exploration and discovery-driven approaches to arrive at new mechanisms and phenomena. This experimental approach is empowering progress, in what are otherwise highly nonlinear, and therefore both nontrivial and counterintuitive, systems. We have found the following experimental techniques to be particularly enabling in this framework: (i) casting with silicone-based elastomers to fabricate samples, (ii) 3D printing, (iii) digital imaging, and (iv) 3D scanning. In particular, digital fabrication and rapid prototyping techniques offer the opportunity to fabricate organic morphable specimens with an unprecedented level of flexibility, in both geometry and materials. Moreover, digital imaging (high-resolution/speed and 3D-scanning) enables accurate quantification of the geometric configurations in the postbuckling regime that can be a powerful tool for discovery and in the detailed validation of models.

There are also significant opportunities in numerical and theoretical analysis that revive results from the classic mechanics literature into new contexts and call for new developments. Even if a detailed review of these opportunities is beyond the scope of this perspective, we pick on two specific aspects. First, the nonlinear nature of the complex geometries that is encountered in buckliphilia make the derivation of closed analytical solutions a challenging, and sometimes unachievable, endeavor. As an alternative, approximate solutions and scaling laws obtained from an energy balance of the primary ingredients assume a new level of importance. These scalings help systematize physical understanding and provide design guidelines that can facilitate implementation by nonspecialists.

Second, on the topic of computer simulations, the mechanics of slender structures, especially in their postbuckling regimes, is pushing the limits of well-established numerical techniques, such as the finite element method (FEM). Accurately reproducing the resulting geometric nonlinearities using FEM is challenging and computationally costly due to both poor convergence and the existence of many neighboring buckling modes. To deal with the first, we have greatly benefited from porting cutting-edge algorithms from the computer graphics community that exploit the power of discrete differential geometry for geometrically exact, efficient and robust computation. For example, using the discrete elastic rod method [26], we have been able to predictively compute the full phase diagram of the nonlinear coiling patterns that emerge when a thin elastic rod is deployed onto a rigid moving substrate $[27,28]$. Moreover, multistability is often ubiquitous in these systems due to the presence of many neighboring modes that can interact nonlinearly. One way to address this issue of multistable solutions is to turn to numerical continuation techniques, as we have illustrated for thin elastic rods [29-31] using the asymptotic numerical method [32]. We believe that translating approaches from computer graphics into engineering as predictive tools and developing new computational techniques offer tremendous opportunities for engineering mechanics.

In summary, the recent revival in the study of mechanical instabilities of slender structures, is opening exciting new research directions that are fundamentally challenging and offer unprecedented opportunities for applications. The post-buckling regime allows for dramatic reconfigurations that can be exploited for function. The prominence of geometry in this class of problems leads to a scale-invariance and universality that confers relevance over a wide range of length-scales. There has been a striking dynamism in this area, which has come to be warmly referred to by some as the Extreme Mechanics movement [33]. This upsurge in activity is demonstrated by the many symposia and focus sessions at leading conferences; e.g., the Society of Engineering Science, the American Society of Mechanical Engineers, and the American Physical Society. This activity is attracting young and well-established researchers, alike, who are blurring disciplinary boundaries with important contributions coming across mechanics, physics, materials science, chemistry, mathematics, and computer science.

Looking forward, some of the exciting opportunities ahead include accurately addressing issues of self-contact and coupling geometrically nonlinear mechanics with other phenomena; e.g., adhesion, growth, fracture, flow, and electromagnetism. As we continue to push ahead, it is important that wheels are not re-invented but, instead, that we revive the well-established mechanics literature respectfully, and continue to advance its legacy into fundamentally new grounds, while remaining open to a diversity of approaches. Simultaneously, it is important that we maintain a good balance between a curiosity-driven research and a drive for innovative engineering. "The future is mechanics" [34], as one of our colleagues often says, and some of it is both slender and geometrically nonlinear.

\section{Acknowledgment}

The author was grateful for financial support from the National Science Foundation: CMMI-1129894 and CMMI-1351449 Faculty Early Career Development (CAREER) Program. We thank Eitan Grinspun, with whom the author came up with the allegory of buckliphobia versus buckliphilia and to Anna Lee, Joel Marthelot, and Francisco López Jiménez for a critical read of the manuscript.

\section{References}

[1] Euler, L., 1744, Methodus Inveniendi Lineas Curvas Maximi Minimive Porprietate Gaudentes (Appendix, De Curvis Elasticis), Marcum Michaelem Bosquet, Lausanne, Switzerland.

2] Timoshenko, S., 1953, History of Strength of Materials: With a Brief Accoun of the History of Theory of Elasticity and Theory of Structures, Dover Publications, Mineola, NY.

[3] Bažant, Z. and Cedolin, L., 2010, Stability of Structures: Elastic, Inelastic, Fracture and Damage Theories, World Scientific, Singapore.

[4] Bažant, Z., 2000, “Structural Stability,” Int. J. Solids Struct., 37(1), pp. 55-67.

[5] Amar, M., and Pomeau, Y., 1997, "Crumpled Paper," Proc. R. Soc. London A, 453(1959), pp. 729-755.

[6] Cerda, E., and Mahadevan, L., 1998, "Conical Surfaces and Crescent Singularities in Crumpled Sheets," Phys. Rev. Lett., 80(11), p. 2358

[7] Lobkovsky, A. E., and Witten, T. A., 1997, "Properties of Ridges in Elastic Membranes," Phys. Rev. E, 55(2), p. 1577.

[8] Blair, D., and Kudrolli, A., 2005, "Geometry of Crumpled Paper," Phys. Rev. Lett., 94(16), p. 166107

[9] Mao, S., Wen, Z., Kim, H., Lu, G., Hurley, P., and Chen, J., 2012, “A General Approach to One-Pot Fabrication of Crumpled Graphene-Based Nanohybrids for Energy Applications," ACS Nano, 6(8), pp. 7505-7513.

[10] El Rouby, W., 2015, "Crumpled Graphene: Preparation and Applications," R. Soc. Chem. Adv., 5(82), pp. 66,767-66,796.

[11] Blees, M., Barnard, A., Rose, P., Roberts, S., McGill, K., Huang, P., Ruyack, A., Kevek, J., Kobrin, B., Muller, D., and McEuen, P., 2015, "Graphene Kirigami," Nature, 524(7564), pp. 204-207.

[12] Miller, J., Su, T., Pabon, J., Wicks, N., Bertoldi, K., and Reis, P., 2015 "Buckling of a Thin Elastic Rod Inside a Horizontal Cylindrical Constraint," Extreme Mech. Lett., 3, pp. 36-44. 
[13] Miller, J., Mulcahy, C., Pabon, J., Wicks, N., and Reis, P., 2015, "Extending the Reach of a Rod Injected Into a Cylinder Through Distributed Vibration," ASME J. Appl. Mech., 82(2), p. 021003.

[14] Miller, J., Su, T., Pabon, J., Wicks, N., Bertoldi, K., and Reis, P., 2015, "Buckling-Induced Lock-up of a Slender Rod Injected Into a Horizontal Cylinder," Int. J. Solids Struct., 72, pp. 153-164.

[15] Hu, N., and Burgueño, R., 2015, "Buckling-Induced Smart Applications: Recent Advances and Trends," Smart Mater. Struct., 24(6), p. 063001.

[16] Kota, S., 2014, "Shape-Shifting Things to Come," Sci. Am., 310(5), pp. 58-65.

[17] Terwagne, D., Brojan, M., and Reis, P., 2014, "Smart Morphable Surfaces for Aerodynamic Drag Control," Adv. Mater., 26(38), pp. 6608-6611.

[18] Brojan, M., Terwagne, D., Lagrange, R., and Reis, P., 2015, "Wrinkling Crystallography on Spherical Surfaces," Proc. Natl. Acad. Sci. U.S.A., 112(1), pp. 14-19.

[19] Stoop, N., Lagrange, R., Terwagne, D., Reis, P. M., and Dunkel, J., 2015, "Curvature-Induced Symmetry Breaking Determines Elastic Surface Patterns," Nat. Mater., 14(3), pp. 337-342.

[20] Shim, J., Perdigou, C., Chen, E., Bertoldi, K., and Reis, P., 2012, "BucklingInduced Encapsulation of Structured Elastic Shells Under Pressure," Proc. Natl. Acad. Sci. U.S.A., 109(16), pp. 5978-5983.

[21] Bertoldi, K., Reis, P., Willshaw, S., and Mullin, T., 2010, "Negative Poisson's Ratio Behavior Induced by an Elastic Instability," Adv. Mat., 22(3), pp. 361-366.

[22] Lazarus, A., and Reis, P., 2015, "Soft Actuation of Structured Cylinders Through Auxetic Behavior," Adv. Eng. Mater., 17(6), pp. 815-820.

[23] Xu, S., Yan, Z., Jang, K.-I., Huang, W., Fu, H., Kim, J., Wei, Z., Flavin, M., McCracken, J., Wang, R., Badea, A., Liu, Y., Xiao, D., Zhou, G., Lee, J., Chung, H. U., Cheng, H., Ren, W., Banks, A., Li, X., Paik, U., Nuzzo, R. G.,
Huang, Y., Zhang, Y., and Rogers, J. A., 2015. "Assembly of Micro/ Nanomaterials Into Complex, Three-Dimensional Architectures by Compressive Buckling," Science, 347(6218), pp. 154-159.

[24] Son, K., Guasto, J., and Stocker, R., 2013, "Bacteria Can Exploit a Flagellar Buckling Instability to Change Direction," Nat. Phys., 9(8), pp. 494-498.

[25] Jawed, M., Khouri, N., Da, F., Grinspun, E., and Reis, P., 2015, "Propulsion and Instability of a Flexible Helical Rod Rotating in a Viscous Fluid," Phys. Rev. Lett. (in press); arXiv:1509.05373 [cond-mat.soft].

[26] Bergou, M., Wardetzky, M., Robinson, S., Audoly, B., and Grinspun, E., 2008, "Discrete Elastic Rods," ACM Trans. Graph., 27(3), p. 63.

[27] Jawed, M., Da, F., Joo, J., Grinspun, E., and Reis, P., 2014, "Coiling of Elastic Rods on Rigid Substrates," Proc. Natl. Acad. Sci., 111(41), pp. $14,663-14,668$.

[28] Jawed, M., and Reis, P., 2014, "Pattern Morphology in the Elastic Sewing Machine," Extreme Mech. Lett., 1, pp. 76-82.

[29] Lazarus, A., Miller, J., and Reis, P., 2013, "Continuation of Equilibria and Stability of Slender Elastic Rods Using an Asymptotic Numerical Method," J. Mech. Phys. Solids, 61(8), pp. 1712-1736.

[30] Lazarus, A., Miller, J., Metlitz, M., and Reis, P., 2013, "Contorting a Heavy and Naturally Curved Elastic Rod," Soft Matter, 9(34), pp. 8274-8281.

[31] Miller, J., Lazarus, A., Audoly, B., and Reis, P., 2014, "Shapes of a Suspended Curly Hair," Phys. Rev. Lett., 112(6), p. 068103.

[32] Cochelin, B., 1994, "A Path-Following Technique Via an AsymptoticNumerical Method,” Comput. Struct., 53(5), pp. 1181-1192.

[33] Krieger, K., 2012, "Extreme Mechanics: Buckling Down," Nature, 488(7410), pp. 146-147.

[34] Huang, Y., 2014, private communication. 